\title{
Spectral Efficiency of Distributed Large-scale MIMO Systems with ZF Receivers
}

DOI:

10.1109/TVT.2016.2616173

Document Version

Accepted author manuscript

Link to publication record in Manchester Research Explorer

\section{Citation for published version (APA):}

Almelah, H., \& Hamdi, K. (2016). Spectral Efficiency of Distributed Large-scale MIMO Systems with ZF Receivers. IEEE Transactions on Vehicular Technology, pp(99). https://doi.org/10.1109/TVT.2016.2616173

\section{Published in:}

IEEE Transactions on Vehicular Technology

\section{Citing this paper}

Please note that where the full-text provided on Manchester Research Explorer is the Author Accepted Manuscript or Proof version this may differ from the final Published version. If citing, it is advised that you check and use the publisher's definitive version.

\section{General rights}

Copyright and moral rights for the publications made accessible in the Research Explorer are retained by the authors and/or other copyright owners and it is a condition of accessing publications that users recognise and abide by the legal requirements associated with these rights.

\section{Takedown policy}

If you believe that this document breaches copyright please refer to the University of Manchester's Takedown Procedures [http://man.ac.uk/04Y6Bo] or contact uml.scholarlycommunications@manchester.ac.uk providing relevant details, so we can investigate your claim.

\section{OPEN ACCESS}




\title{
Spectral Efficiency of Distributed Large-scale MIMO Systems with ZF Receivers
}

\author{
Hisham B. Almelah, and Khairi Ashour Hamdi
}

\begin{abstract}
Distributed multiple-input multiple-output (DMIMO) is a promising technique for next generation wireless networks, which offers a remarkable spectral efficiency gain over the conventional co-located MIMO (C-MIMO). In contrast to $\mathrm{C}$ MIMO, which can be regarded as a special case of D-MIMO, performance analysis of D-MIMO is a challenging problem. This is because radio channels between a user and the distributed radio ports (RPs) are characterized by non-identical path-loss and shadowing effects which render the classical analytical methods non-tractable. In this paper, new accurate expressions for the uplink spectral efficiency of D-MIMO and C-MIMO systems are presented and compared for given large-scale coefficients. We further consider the uplink spectral efficiency for a single-cell distributed large-scale MIMO (D-LMIMO) system with linear zero-forcing (ZF) receivers, accounting for path loss along with shadow fading and multi-path fading effects. Exact expressions for the average spectral efficiency over shadow fading in the asymptotically very large number of RPs antennas regime is explicitly derived, and a tight closed-form lower bound on the asymptotic spectral efficiency is presented. We demonstrate that, the transmit power of each user in D-LMIMO can be scaled down proportionally to the inverse of the number of RP antennas with no performance reduction. Moreover, we study the spectral efficiency of a D-MIMO in a multi-cell environment taking into account accurate co-channel interference (CCI) models. These expressions provide meaningful insights into the impact of SNR, RPs and user positions, number of RPs antennas, shadow fading, and out-of-cell interference on the spectral efficiency of D-MIMO over practical scenarios. Finally, numerical results are validated by simulation to confirm our analysis.
\end{abstract}

Index Terms-Distributed antenna system (DAS), multipleinput multiple-output (MIMO) systems, large-scale MIMO (LMIMO), spectral efficiency, zero-forcing (ZF) receiver, co-channel interference (CCI).

\section{INTRODUCTION}

M ULTIPLE-INPUT multiple-output (MIMO) technology, which relies on multiple antennas to transmit multiple data streams simultaneously in wireless systems, can offer much higher data rates to a large number of users compared with single-input single-output (SISO) systems without the need to increase the transmit power or bandwidth. In recent years, the performance of MIMO technology has significantly attracted research interest [1]-[8].

Distributed antenna system (DAS) has been originally implemented to cover dead spots in indoor wireless communications and then used to maximize cell coverage in cellular networks [9]-[12]. This setting has recently gained a great deal of interest as a promising potential to satisfy fast-growing demands for next generation wireless systems because of

The authors are with the school of Electrical and Electronic Engineering, The University of Manchester, Manchester, M13 9PL, UK. (e-mails: hisham.almelah@postgrad.manchester.ac.uk, k.hamdi@manchester.ac.uk). its power savings and capacity merit over conventional colocated antenna system (CAS) [13]-[18]. In DAS, radio ports (RPs) are geographically distributed over a cell to reduce access distance along with the transmit power which results in mitigating the co-channel interference [19]-[22]. Each radio port is connected to a central processing unit (PU) by fiber or exclusive wireless link.

Combining the conventional point-to-point MIMO technology with the basic principle of DAS introduced the socalled distributed MIMO (D-MIMO) system which takes the advantages of both spatial multiplexing and macro-diversity [23]-[25]. However, as a result of different propagation paths of a user to different distributed antennas, each path subjects to independent and different degree of large-scale fading effects (i.e., path loss along with shadowing fading). This makes the theoretical performance analysis over D-MIMO channel more complicated. As such, only a few analytical studies (e.g., [26][29]) have investigated the performance of D-MIMO systems with an optimal nonlinear receivers over a composite fading channel which includes both small and large-scale fading.

In [26], an accurate approximation of the outage capacity and upper bound on the achievable capacity were determined for D-MIMO system in a composite fading channel. Employing the high signal-to-noise ratio (SNR) approximation, the downlink ergodic capacity of D-MIMO channel including path loss, shadow fading and multi-path fading has been extensively investigated in [27]. For the uplink scenario, the authors of [28] presented and compared the spectral efficiency for D-MIMO system and co-located MIMO (C-MIMO) system over a composite fading channel at high SNR, whereas the authors in [29] approximated the composite Gamma-Gamma distribution for a composite fading channel by a Gamma distribution, accordingly they proposed an approximate upper bound on the spectral efficiency of D-MIMO system.

In this context, we mainly focus on D-MIMO system with linear zero-forcing $(\mathrm{ZF})$ receivers. $\mathrm{ZF}$ receivers induce low complexity and implementation cost compared to nonpractical receivers which were considered in [26]-[29]. In a recent publication [30], the performance of distributed largescale MIMO (D-LMIMO) systems with ZF over only smallscale fading (i.e., path loss and shadowing were ignored) was reported, wherein the authors studied the uplink of a distributed large-scale MIMO system with large number of single-antenna RPs relative to the number of single-antenna users. To the best of our knowledge, most studies published regarding MIMO systems with ZF were only over multi-path fading (e.g., [31]). Only studies dealing with the performance analysis of ZF over a composite MIMO channel models were conducted in [32]-[35]. In [32], the spectral efficiency 
of C-MIMO system with ZF receiver was examined over Rayleigh/lognormal (RLN) fading channel. The analysis was limited to C-MIMO system and the final result is not amenable to further manipulation. In [33], the authors presented an approximation to compute the downlink spectral efficiency as a function of user location for D-MIMO with zero-forcing beamforming under high SNR conditions. Shadowing effects and large-scale MIMO were not considered in [33] whereas the authors in [34] and [35] investigated the performance of $\mathrm{ZF}$ receivers in D-MIMO systems, in which wireless links were assumed between RPs and PU which acts as a base station (BS), making these models theoretically quite similar to co-located multi-user MIMO (MU-MIMO) systems. Based on a set of empirical data, [34] approximated the log-normal shadowing model by the analytically friendlier Gamma model, as well as the performance of D-LMIMO system was not considered. In [35], closed-form upper and lower bounds on the spectral efficiency were derived over a classical RLN fading model and used to provide the spectral efficiency of D-LMIMO system.

This paper presents a new exact analysis of the uplink spectral efficiency of a single user equipped with $N_{t}$ transmit antennas in a single-cell D-LMIMO system operating over a composite fading channel in the presence of ZF linear receiver at PU, which can be regarded as BS. This system also consists of a number of RPs connected with PU via optical fibers, each of them equipped with $N_{r}$ receive antennas. We further shed the light on the effect of co-channel interference (CCI) on the spectral efficiency of the system. In particular, the contributions of the paper can be summarized as follows:

- It is known that, there are difficulties in analyzing the spectral efficiency of D-MIMO system with ZF receivers where the overall SNR involves a sum of non-identical Wishart matrices instead of just one in C-MIMO systems with $\mathrm{ZF}$ receivers, making the performance analysis of this model more challenging. Using the mathematical results in [36], the sum of non-identical Wishart matrices is well approximated by a single Wishart matrix and an accurate approximation of PDF of the instantaneous SNR at the output of the ZF receiver [37] in D-MIMO system is obtained. The new approximation is more accurate than the approximation in [31, Eq. (12)].

- Based on the above, an accurate analytical expression for the average spectral efficiency over small-scale fading in D-MIMO system with ZF receiver is analytically derived. This expression of the spectral efficiency can be easily simplified to a finite summation using the numerical quadrature methods for common distributions. Theoretical comparisons between C-MIMO system and a specific setting of D-MIMO system are also provided for finite number of receive antennas, which reveal the performance improvement of spectral efficiency of DMIMO system over C-MIMO system. Moreover, with the aid of the expressions obtained, numerical results for a specific setting of D-MIMO system reveals the optimum sites of RPs, which result in maximizing the spectral efficiency. It is also shown when $N_{r}>N_{t}$, the analytical results accurately match the simulation results.

- Through averaging the spectral efficiency over shadow fading in the asymptotically large number of RPs antennas regime (i.e., D-LMIMO system), we explicitly derive an exact analytical expression for the spectral efficiency, enabling to highlight into the effects of model parameters such as shadowing on the performance of the system. It is shown that, the transmit power of each user can be reduced proportionally to $1 / N_{r}$ while maintaining the same performance. Furthermore, we propose an exact closedform lower bound on the asymptotic spectral efficiency for $N_{r} \rightarrow \infty$.

- In addition, we derive an approximate closed-form analytical expression for the spectral efficiency as a function of large-scale parameters in the presence of the interference from other cells. This expression enables us to study the effect of out-of-cell interference on the system performance.

The rest of this paper is organized as follows: In Section II, the D-MIMO system model under consideration is introduced. In Section III, we present the spectral efficiency formulation of the system with ZF receiver. The spectral efficiency with a finite number of RP antennas is investigated in Section IV. In Section V, we derive a new analytical expression for the spectral efficiency along with lower bound for the system with large number of RP antennas. The spectral efficiency analysis is extended to the multi-cell system in Section VI, where the co-channel interference is included. A set of numerical results is provided in Section VII. Finally, Section VIII concludes the paper.

Notation: Upper and lower case boldface denotes matrices and vectors, respectively, while $[\cdot]_{i j}$ represents the $(i, j)$ th element of the matrix and $[\cdot]_{i}$ denotes the $i$ th row of the matrix. $N \times N$ identity matrix is expressed as $I_{N}$. Superscripts $T, H$, and $\dagger$ stand for transpose, conjugate transpose, and pseudo-inverse, respectively, $\otimes$ denotes Kronecker product, and the symbol $\mathbb{C}$ stands for the set of complex numbers. The expectation operation is expressed via $\mathbb{E}(\cdot)$. Finally, $\mathcal{C N}(\mathbf{0}, \boldsymbol{\Sigma})$ denotes a circularly symmetric complex normal variable vector with zero mean and covariance matrix $\boldsymbol{\Sigma}$.

\section{SYSTEM MODEL}

We consider an uplink single-cell D-MIMO system with $L$ RPs deployed at arbitrary locations, each of which is equipped with $N_{r}$ receive antennas. All receive antennas of RPs are assumed to have a separate feeder to the PU, where all signal processing is achieved, through optical fibers. The system under investigation is depicted in Fig. 1. We assume a single user with $N_{t}$ antennas, and $N_{r} \geq N_{t}$ to enable each RP to serve all antennas simultaneously. Assuming the channel is perfectly known at the receiver and unknown at the transmitter, the available average power, $P$, is equally split between all data streams. This assumption maximizes the worst-case capacity and therefore can provide a lower bound on the performance of practical systems. As such, the overall uplink received signal at the PU is

$$
\boldsymbol{y}=\sqrt{p_{u}} \boldsymbol{G} \boldsymbol{x}+\boldsymbol{n}
$$




\section{ZF ANALYSIS}

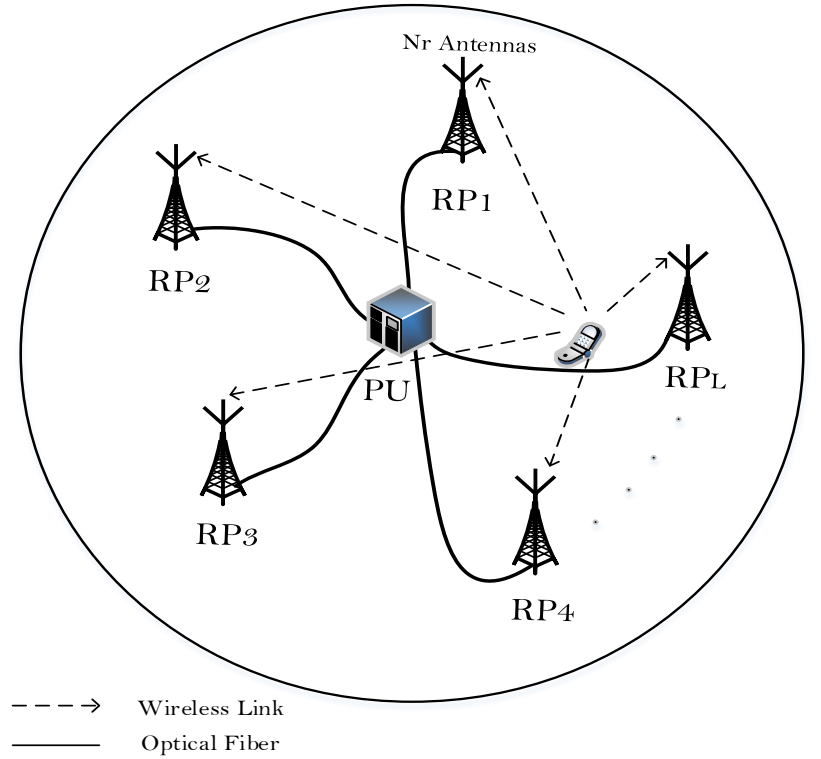

Fig. 1. D-MIMO system model

where $p_{u}=P / N_{t}$ is the average power transmitted by each antenna, $\boldsymbol{y} \in \mathbb{C}^{L N_{r} \times 1}$ is the received signal vector, $\boldsymbol{x} \in \mathbb{C}^{N_{t} \times 1}$ is the transmitted signal vector with $\mathbb{E}\left(\boldsymbol{x} \boldsymbol{x}^{H}\right)=\boldsymbol{I}_{N_{t}}$, and $\boldsymbol{n} \in \mathbb{C}^{L N_{r} \times 1}$ is the complex additive white Gaussian noise (AWGN) vector, such that $\boldsymbol{n} \sim \mathcal{C N}\left(\mathbf{0}, \boldsymbol{I}_{L N_{r}}\right)$. The total channel matrix $\boldsymbol{G} \in \mathbb{C}^{L N_{r} \times N_{t}}$ is composed of $L$ independent subchannel matrices for each RP, $\boldsymbol{G}=\left[\boldsymbol{G}_{1} \boldsymbol{G}_{2} \cdots \boldsymbol{G}_{L}\right]^{T}$ where $\boldsymbol{G}_{l} \in \mathbb{C}^{N_{r} \times N_{t}}(l=1,2 \cdots, L)$ is a channel matrix between the user and the $l$ th RP. It incorporates both independent small and large-scale fading, and is modeled as

$$
\boldsymbol{G}_{l}=\sqrt{\beta_{l}} \boldsymbol{H}_{l}
$$

where $\boldsymbol{H}_{l} \in \mathbb{C}^{N_{r} \times N_{t}}$ represents the small-scale fading coefficients from the user to the lth RP which are assumed to be circularly symmetric complex Gaussian RVs with zero mean and unit variance, and $\beta_{l}$ models the path-loss attenuation and log-normal shadow fading as

$$
\beta_{l}=s_{l}\left(d_{l}\right)^{-v}
$$

where $s_{l}$ represents the log-normal shadow fading, i.e.,

$$
s_{l}(\mathrm{~dB}) \sim \mathcal{N}\left(0, \sigma^{2}\right)
$$

and

$$
s_{l} \sim e^{\varsigma_{l}}
$$

where $\sigma$ is a standard deviation in $\mathrm{dB}, \varsigma_{l} \sim \mathcal{N}\left(0, \lambda^{2} \sigma^{2}\right)$ where $\lambda=\frac{\ln 10}{10}, v \geq 2$ is the path-loss exponent. $d_{l}=\left|\boldsymbol{D}_{l}-\boldsymbol{\rho}\right|$ indicates the distance from the user to the $l$ th RP, where $\boldsymbol{D}_{l}$ and $\rho$ are position vectors to the certain reference point in the coverage area from the $l$ th RP and user, respectively.
By using the ZF linear receiver at the PU which has perfect CSI of all channels between the user and RPs in the respective cell, the intracell interference is totally eliminated, and the received vector $\boldsymbol{y}$ can be separated into streams by multiplying it with pseudo-inverse of $\boldsymbol{G}, \boldsymbol{G}^{\dagger} \triangleq\left(G^{H} \boldsymbol{G}\right)^{-1} \boldsymbol{G}^{H}$, as follows

$$
\boldsymbol{r}=\boldsymbol{G}^{\dagger} \boldsymbol{y}=\sqrt{p_{u}} \boldsymbol{x}+\boldsymbol{G}^{\dagger} \boldsymbol{n} .
$$

Then, the $k$ th element of $\boldsymbol{r}$ is expressed as

$$
r_{k}=\sqrt{p_{u}} x_{k}+\left[\boldsymbol{G}^{\dagger}\right]_{k} \boldsymbol{n}
$$

where $x_{k}$ is the $k$ th element of the vector $\boldsymbol{x}, p_{u}$ is the average SNR. Then, the uplink instantaneous received SNR at the $k$ th ZF output $\left(\gamma_{k}\right)$ is equal to

$$
\begin{aligned}
\gamma_{k}= & \frac{p_{u}}{\left[\left(\boldsymbol{G}^{H} \boldsymbol{G}\right)^{-1}\right]_{k k}} \\
= & \frac{p_{u}}{\left[\left(\boldsymbol{H}^{H}\left(\boldsymbol{\Xi} \otimes \boldsymbol{I}_{N_{r}}\right) \boldsymbol{H}\right)^{-1}\right]_{k k}}
\end{aligned}
$$

where

$$
\boldsymbol{H}=\left[\boldsymbol{H}_{1} \boldsymbol{H}_{2} \cdots \boldsymbol{H}_{L}\right]^{T}
$$

and

$$
\boldsymbol{\Xi}=\operatorname{diag}\left(\beta_{1}, \beta_{2}, \ldots, \beta_{L}\right)
$$

where $\operatorname{diag}($.$) denotes the diagonal matrix.$

If the SNR at the cell boundary is taken as a reference SNR, equation (8) is rewritten as

$$
\gamma_{k}=\frac{\gamma}{\left[\left(\boldsymbol{H}^{H}\left(\boldsymbol{\Xi} \otimes \boldsymbol{I}_{N_{r}}\right) \boldsymbol{H}\right)^{-1}\right]_{k k}}
$$

where

$$
\gamma=p_{u}(D)^{-v}
$$

and

$$
\boldsymbol{\Xi}=\operatorname{diag}\left[s_{1}\left(\frac{d_{1}}{D}\right)^{-v} s_{2}\left(\frac{d_{2}}{D}\right)^{-v} \cdots s_{L}\left(\frac{d_{L}}{D}\right)^{-v}\right] .
$$

Then, the uplink instantaneous spectral efficiency in $\mathrm{bits} / \mathrm{s} / \mathrm{Hz}$ is given by

$$
\mathcal{R}(\boldsymbol{H}, \boldsymbol{\Xi})=\sum_{k=1}^{N_{t}} \log _{2}\left(1+\gamma_{k}\right) .
$$

Averaging (14) over $\boldsymbol{H}$ (i.e., small-scale fading), we have

$$
\mathcal{R}(\boldsymbol{\Xi})=\sum_{k=1}^{N_{t}} \mathbb{E}_{\boldsymbol{H}}\left[\log _{2}\left(1+\gamma_{k}\right)\right] .
$$

where the expectation is taken over all channel realizations of $\boldsymbol{H}$. 


\section{FInITE- $N_{r}$ ANALYSis}

In this section, we provide an approximate closed-form expression for the average spectral efficiency of the system for given large-scale coefficients.

Theorem 1. For given large-scale coefficients, $\gamma_{k}$ in (8) can be represented as

$$
\begin{aligned}
\gamma_{k}= & \frac{p_{u}}{\left[\left(\sum_{l=1}^{L} \boldsymbol{G}_{l}^{H} \boldsymbol{G}_{l}\right)^{-1}\right]_{k k}} \\
= & \frac{p_{u}}{\left[\left(\sum_{l=1}^{L} \beta_{l} \boldsymbol{H}_{l}^{H} \boldsymbol{H}_{l}\right)^{-1}\right]_{k k}} \approx p_{u} X_{k}
\end{aligned}
$$

where

$$
\begin{gathered}
X_{k} \sim \operatorname{Gamma}\left(\theta_{f}, \Theta\right) \\
\theta_{f}=N_{r}\left(\sum_{l=1}^{L} \beta_{l}\right)^{2} /\left(\sum_{l=1}^{L} \beta_{l}^{2}\right)-N_{t}+1
\end{gathered}
$$

and

$$
\Theta=[\boldsymbol{\Sigma}]_{k k}=\left(\sum_{l=1}^{L} \beta_{l}^{2}\right) /\left(\sum_{l=1}^{L} \beta_{l}\right) .
$$

Proof: Since $\boldsymbol{G}_{l}^{H} \boldsymbol{G}_{l}$ is a complex central Wishart matrix [37] with degrees of freedom $N_{r}$ and covariance matrix $\boldsymbol{\Sigma}_{l}=\beta_{l} \boldsymbol{I}_{N_{t}}$, the matrix $\sum_{l=1}^{L} \boldsymbol{G}_{l}^{H} \boldsymbol{G}_{l}$ has approximately a complex central Wishart distribution with degrees of free$\operatorname{dom} N_{r}\left(\sum_{l=1}^{L} \beta_{l}\right)^{2} /\left(\sum_{l=1}^{L} \beta_{l}^{2}\right)$ and covariance matrix $\boldsymbol{\Sigma}=$ $\left(\sum_{l=1}^{L} \beta_{l}^{2}\right) /\left(\sum_{l=1}^{L} \beta_{l}\right) \boldsymbol{I}_{N_{t}}$ [36].

Based on a result in [37], we have $\gamma_{k}$, given in (16).

In the case of C-MIMO channel (i.e. $L=1$ ), $\boldsymbol{G}_{1}^{H} \boldsymbol{G}_{1}$ has exactly a complex central Wishart distribution with $N_{r}$ degrees of freedom and covariance matrix $\boldsymbol{\Sigma}=\beta_{1} \boldsymbol{I}_{N_{t}}$, resulting in $X_{k} \sim \operatorname{Gamma}\left(N_{r}-N_{t}+1, \beta_{1}\right)$.

For convenience of analysis, we use the following lemma to exactly obtain the required average in (14).

Lemma 1. [39, Eq. (6)] For any $x>0$

$$
\ln (1+x)=\int_{0}^{\infty} \frac{1}{z}\left(1-e^{-x z}\right) e^{-z} d z
$$

Theorem 2. An approximate closed-form analytical expression for the average spectral efficiency of D-MIMO system with ZF receivers over small-scale fading is given by

$$
\mathcal{R}\left(\beta_{l}\right)=N_{t}\left(\log _{2} e\right) \sum_{i=1}^{I} \frac{\mathcal{A}_{i}}{\mathcal{B}_{i}}\left(1-\mathcal{M}\left(\beta_{l}\right)\right)+R_{I}
$$

where $\mathcal{M}\left(\beta_{l}\right)=\left(\frac{1}{1+\mathcal{C}_{i} \Theta}\right)^{\theta_{f}}, \mathcal{C}_{i}=p_{u} \mathcal{B}_{i}, \mathcal{A}_{i}$ and $\mathcal{B}_{i}$ are the $i$ th weight and abscissa, respectively, of the $I$ th order Laguerre polynomial, tabulated in [41, Eq. (25.4.45)]. The remainder $R_{I}$ is negligible for $I \geq 15$.
Proof: From Lemma 1, an exact analytical expression for the average spectral efficiency over small-scale fading is determined as follows

$$
\begin{aligned}
\mathcal{R}\left(\beta_{l}\right)= & \sum_{k=1}^{N_{t}} \mathbb{E}_{X_{k}}\left[\left(\log _{2} e\right) \int_{0}^{\infty} \frac{e^{-z / p_{u}}}{z}\left(1-e^{-z X_{k}}\right) d z\right] \\
& =\log _{2} e \sum_{k=1}^{N_{t}} \int_{0}^{\infty} \frac{e^{-z / p_{u}}}{z}\left(1-\mathcal{M}\left(z, \beta_{l}\right)\right) d z \\
& =N_{t} \log _{2} e \int_{0}^{\infty} \frac{e^{-z / p_{u}}}{z}\left(1-\mathcal{M}\left(z, \beta_{l}\right)\right) d z
\end{aligned}
$$

where $\mathcal{M}\left(z, \beta_{l}\right)=\mathbb{E}_{X_{k}}\left[e^{-z X_{k}}\right]$.

From Theorem $1, \mathcal{M}\left(z, \beta_{l}\right)$ is expressed as

$$
\mathcal{M}\left(z, \beta_{l}\right)=\left(\frac{1}{1+z \Theta}\right)^{\theta_{f}} .
$$

Substituting (23) into (22), we have

$$
\mathcal{R}\left(\beta_{l}\right)=N_{t}\left(\log _{2} e\right) \int_{0}^{\infty} \frac{e^{-z / p_{u}}}{z}\left(1-\left(\frac{1}{1+z \Theta}\right)^{\theta_{f}}\right) d z .
$$

Applying the Gauss-Laguerre quadrature rule on (24), we conclude the proof.

Assuming $\beta_{l}=\left(\frac{d_{l}}{D}\right)^{-v}$ to account for the effects of path-loss attenuation, the average spectral efficiency in (21) becomes

$$
\mathcal{R}\left(d_{l}\right)=N_{t}\left(\log _{2} e\right) \sum_{i=1}^{I} \frac{\mathcal{A}_{i}}{\mathcal{B}_{i}}\left(1-\mathcal{M}\left(d_{l}\right)\right)+R_{I}
$$

where

$$
\Theta=\frac{\sum_{l=1}^{L}\left(\frac{d_{l}}{D}\right)^{-2 v}}{\sum_{l=1}^{L}\left(\frac{d_{l}}{D}\right)^{-v}}
$$

and

$$
\theta_{f}=\frac{N_{r}\left(\sum_{l=1}^{L}\left(\frac{d_{l}}{D}\right)^{-v}\right)^{2}}{\sum_{l=1}^{L}\left(\frac{d_{l}}{D}\right)^{-2 v}}-N_{t}+1 .
$$

The average spectral efficiency with respect to $d_{l}$ can be then calculated by averaging the $\mathcal{R}\left(d_{l}\right)$ in (25) over all possible user locations.

\section{Asymptotic $\left(N_{r} \rightarrow \infty\right)$ Analysis}

Recently, there has been increasing research interest in CMIMO systems with a very large of BS antennas, which promise to improve the system performance with significant power savings. As it is well known, the small-scale fading can be averaged out by deploying a very large of antennas at BSs. Here, we elaborate on analyzing the impact of large-scale fading on the spectral efficiency of large D-MIMO systems with ZF receivers. To guarantee the total received at each RP does not diverge as $N_{r} \rightarrow \infty$, we should normalize 
the transmit power by the large number of RP antennas $N_{r}$. Consequently, $\gamma_{k}$ can be re-expressed as

$$
\begin{aligned}
\gamma_{k}= & \frac{\gamma}{N_{r}\left[\left(\boldsymbol{H}^{H}\left(\boldsymbol{\Xi} \otimes \boldsymbol{I}_{N_{r}}\right) \boldsymbol{H}\right)^{-1}\right]_{k k}} \\
& =\frac{\gamma}{\left[\left(\sum_{l=1}^{L} s_{l}\left(\frac{d_{l}}{D}\right)^{-v} \frac{\boldsymbol{H}_{l}^{H} \boldsymbol{H}_{l}}{N_{r}}\right)^{-1}\right]_{k k}} .
\end{aligned}
$$

From the law of large numbers which states that $\lim _{N_{r} \rightarrow \infty} \frac{\boldsymbol{H}_{l}^{H} \boldsymbol{H}_{l}}{N_{r}}=\boldsymbol{I}_{N_{r}}$, we obtain

$$
\gamma_{k}=\gamma \sum_{l=1}^{L}\left(\frac{D}{d_{l}}\right)^{v} s_{l} .
$$

Thus, the average asymptotic spectral efficiency is given by

$$
\mathcal{R}=\sum_{k=1}^{N_{t}} \mathbb{E}\left[\log _{2}\left(1+\gamma \sum_{l=1}^{L}\left(\frac{D}{d_{l}}\right)^{v} s_{l}\right)\right] .
$$

\section{A. Exact Analysis}

The average asymptotic spectral efficiency over $s_{l}$ is given by

$$
\mathcal{R}\left(d_{l}\right)=\sum_{k=1}^{N_{t}} \mathbb{E}_{s_{l}}\left[\log _{2}\left(1+\gamma \sum_{l=1}^{L}\left(\frac{D}{d_{l}}\right)^{v} s_{l}\right)\right] .
$$

An exact closed-form expression for the average asymptotic spectral efficiency with respect to $s_{l}$ is derived as follows

Theorem 3. The average asymptotic spectral efficiency of DLMIMO system with ZF receivers over shadow fading (i.e., for given $d_{l}$ ) is given by

$$
\begin{aligned}
\mathcal{R}\left(d_{l}\right)= & N_{t}\left(\log _{2} e\right) \sum_{i=1}^{I} \frac{\mathcal{A}_{i}}{\mathcal{B}_{i}} \\
& \times\left(1-\frac{1}{\sqrt{\pi}} \prod_{l=1}^{L}\left[\sum _ { m = 1 } ^ { M } \xi _ { m } \operatorname { e x p } \left(-\mathcal{C}_{i}\right.\right.\right. \\
& \left.\left.\left.\times\left(\frac{D}{d_{l}}\right)^{v} e^{\sqrt{2} \lambda \sigma \eta_{m}}\right)+R_{M}\right]\right)+R_{I}
\end{aligned}
$$

where $\mathcal{C}_{i}=\gamma \mathcal{B}_{i}, \xi_{j}$ and $\eta_{j}$ are the $j$ th weight and abscissa, respectively, of the $J$ th order Hermite polynomial, tabulated in [41, Eq. (25.4.46)]. The remainder $R_{M}$ is negligible for $M \geq 15$. get

Proof: Similarly, by applying Lemma 1 on (30), we can

$$
\begin{aligned}
\mathcal{R}\left(d_{l}\right)= & \sum_{k=1}^{N_{t}}\left[\left(\log _{2} e\right) \int_{0}^{\infty} \frac{e^{-z / \gamma}}{z}\right. \\
& \left.\times\left(1-\mathbb{E}_{s_{l}}\left[e^{-z \sum_{l=1}^{L}\left(\frac{D}{d_{l}}\right)^{v} s_{l}}\right]\right) d z\right] \\
& =N_{t}\left(\log _{2} e\right)\left[\int_{0}^{\infty} \frac{e^{-z / \gamma}}{z}\right. \\
& \left.\times\left(1-\prod_{l=1}^{L} \mathcal{N}_{l}\left(z, d_{l}\right)\right) d z\right]
\end{aligned}
$$

where $\mathcal{N}_{l}\left(z, d_{l}\right)=\mathbb{E}_{s_{l}}\left[e^{-z\left(\frac{D}{d_{l}}\right)^{v} s_{l}}\right]$.

Likewise, (33) can be evaluated using Gauss-Laguerre quadrature rule as follows

$$
\mathcal{R}\left(d_{l}\right)=N_{t}\left(\log _{2} e\right) \sum_{i=1}^{I} \frac{\mathcal{A}_{i}}{\mathcal{B}_{i}}\left(1-\prod_{l=1}^{L} \mathcal{N}_{l}\left(d_{l}\right)\right)+R_{I}
$$

where $\mathcal{N}_{l}\left(d_{l}\right)=\mathbb{E}_{s_{l}}\left[e^{-\mathcal{C}_{i}\left(\frac{D}{d_{l}}\right)^{v} s_{l}}\right]$.

$\mathcal{N}_{l}\left(d_{l}\right)$ can be calculated as follows

$$
\begin{aligned}
\mathcal{N}_{l}\left(d_{l}\right)= & \int_{-\infty}^{\infty} \exp \left(-\mathcal{C}_{i}\left(\frac{D}{d_{l}}\right)^{v}\left(e^{\varsigma_{l}}\right)\right) \\
& \times \frac{1}{\sqrt{2 \pi} \lambda \sigma} e^{-\frac{\varsigma_{l}^{2}}{2 \lambda^{2} \sigma^{2}}} d \varsigma_{l} .
\end{aligned}
$$

Further, the integral form in (35) can be accurately evaluated using Gauss-Hermite Quadrature rule as

$$
\begin{aligned}
\mathcal{N}_{l}\left(d_{l}\right)= & \frac{1}{\sqrt{\pi}} \sum_{m=1}^{M} \xi_{m} \exp \left(-\mathcal{C}_{i}\right. \\
& \left.\times\left(\frac{D}{d_{l}}\right)^{v} e^{\sqrt{2} \lambda \sigma \eta_{m}}\right)+R_{M} .
\end{aligned}
$$

Finally, substituting (36) into (34) we can infer the proof.

In a similar way, the average asymptotic spectral efficiency over $d_{l}$ can be calculated by averaging the spectral efficiency in (32) over user positions.

\section{B. Closed-Form Lower Bound on the Asymptotic Spectral Efficiency}

In the following, we derive a new explicit tight lower bound on the asymptotic spectral efficiency of the respective system to complement the previous exact analysis and extract interesting insights on the effect of the system parameters.

Theorem 4. The lower bound $\left(\mathcal{R}_{L}\right)$ on the asymptotic spectral efficiency for this system is defined as

$$
\mathcal{R}_{L}=\sum_{k=1}^{N_{t}} \log _{2}\left(1+\gamma e^{v / 2} \sum_{l=1}^{L} e^{\left(-\frac{v D_{l}^{2}}{2 D^{2}}\right)}\right) .
$$

Proof: Recalling that $\log _{2}\left(1+\sum_{i=1}^{m} \alpha_{i} \exp \left(x_{i}\right)\right)$ is convex in $x_{i}$ for $\alpha_{i}>0$, and applying Jensen's inequality, we can lower bound $\mathcal{R}$ in (30) as follows

$$
\mathcal{R} \geq \mathcal{R}_{L}=\sum_{k=1}^{N_{t}} \log _{2}\left(1+\gamma \sum_{l=1}^{L} e^{\mathbb{E}\left[\ln \left(\frac{D}{d_{l}}\right)^{v} s_{l}\right]}\right) .
$$

At a specific user position, we obtain

$$
\mathcal{R}_{L}\left(d_{l}\right)=\sum_{k=1}^{N_{t}} \log _{2}\left(1+\gamma \sum_{l=1}^{L}\left(\frac{D}{d_{l}}\right)^{v} e^{\mathbb{E}_{s_{l}}\left[\ln \left(s_{l}\right)\right]}\right) .
$$

Because $\ln \left(s_{l}\right)$ has zero mean, we have

$$
\mathcal{R}_{L}\left(d_{l}\right)=\sum_{k=1}^{N_{t}} \log _{2}\left(1+\gamma \sum_{l=1}^{L}\left(\frac{D}{d_{l}}\right)^{v}\right) .
$$


On a similar basis, we can lower bound the result in (40) over user locations as

$$
\mathcal{R}_{L}=\sum_{k=1}^{N_{t}} \log _{2}\left(1+\gamma \sum_{l=1}^{L} e^{\mathbb{E}_{d_{l}}\left[\ln \left(\frac{D}{d_{l}}\right)^{v}\right]}\right)
$$

Then, $\mathbb{E}_{d_{l}}\left[\ln \left(\frac{D}{d_{l}}\right)^{v}\right]$ is computed as [28]

$$
\mathbb{E}_{d_{l}}\left[\ln \left(\frac{D}{d_{l}}\right)^{v}\right]=\frac{v}{2}-\frac{v D_{l}^{2}}{2 D^{2}} .
$$

Substituting (42) into (41), we conclude the proof.

\section{Multi-Cell D-MiMO Model}

In this section, we consider a multi-cell D-MIMO model with $J$ cells sharing the same frequency band. Each cell is identical to the cell described in Section II. Then, the overall received vector at the $j$ th $\mathrm{PU}$ is

$$
\begin{aligned}
\boldsymbol{y}_{j}= & \sqrt{p_{u}} \sum_{i=1}^{J} \boldsymbol{G}_{j i} \boldsymbol{x}_{i}+\boldsymbol{n}_{j} \\
= & \sqrt{p_{u}} \boldsymbol{G}_{j j} \boldsymbol{x}_{j}+\sqrt{p_{u}} \sum_{i \neq j}^{J} \boldsymbol{G}_{j i} \boldsymbol{x}_{i}+\boldsymbol{n}_{j}
\end{aligned}
$$

where $\boldsymbol{x}_{i} \in \mathbb{C}^{N_{t} \times 1}$ is the transmitted signal of the user in the $i$ th cell, such that $\mathbb{E}\left(\boldsymbol{x} \boldsymbol{x}^{H}\right)=\boldsymbol{I}_{N_{t}}$, and $\boldsymbol{n}_{j} \in \mathbb{C}^{L N_{r} \times 1}$ is the complex additive white Gaussian noise (AWGN) vector, such that $\boldsymbol{n} \sim \mathcal{C N}\left(\mathbf{0}, \boldsymbol{I}_{L N_{r}}\right)$. With this assumption, $p_{u}$ represents the normalized transmit SNR. $\boldsymbol{G}_{j i} \in \mathbb{C}^{L N_{r} \times N_{t}}$ is the total channel matrix between the user in the $i$ th cell and RPs in the $j$ th cell, $\boldsymbol{G}_{j i}=\left[\boldsymbol{G}_{j i 1} \boldsymbol{G}_{j i 2} \cdots \boldsymbol{G}_{j i L}\right]^{T}$ where $\boldsymbol{G}_{j i l} \in \mathbb{C}^{N_{r} \times N_{t}}$ $(l=1,2 \cdots, L)$ is a channel matrix between the user in the $i$ th cell and the $l$ th RP in the $j$ th cell. It includes both independent small and large-scale fading, and is modeled as

$$
\boldsymbol{G}_{j i l}=\sqrt{\beta_{i l}} \boldsymbol{H}_{j i l}
$$

where $\boldsymbol{H}_{j i l} \in \mathbb{C}^{N_{r} \times N_{t}}$ represents the small-scale fading coefficients from the user in the $i$ th cell to the $l$ th RP in the $j$ th cell, being assumed to be circularly symmetric complex Gaussian $\mathrm{RVs}$ with zero mean and unit variance, and $\beta_{i l}$ models the path-loss attenuation and log-normal shadow fading from the user in the $i$ th cell to the $l$ th RP in the $j$ th cell .

By using the $\mathrm{ZF}$ linear receiver at the $j$ th PU, the intracell interference is totally eliminated, and the received vector $\boldsymbol{y}_{j}$ is separated into streams by multiplying it with pseudo-inverse of $\boldsymbol{G}_{j j}, \boldsymbol{G}_{j j}^{\dagger} \triangleq\left(\boldsymbol{G}_{j j}^{H} \boldsymbol{G}_{j j}\right)^{-1} \boldsymbol{G}_{j j}^{H}$, as follows

$$
\boldsymbol{r}_{j}=\boldsymbol{G}_{j j}^{\dagger} \boldsymbol{y}_{j}=\sqrt{p_{u}} \boldsymbol{x}_{j}+\sqrt{p_{u}} \sum_{i \neq j}^{J} \boldsymbol{G}_{j j}^{\dagger} \boldsymbol{G}_{j i} \boldsymbol{x}_{i}+\boldsymbol{G}_{j j}^{\dagger} \boldsymbol{n}_{j}
$$

Then, the $k$ th element of $\boldsymbol{r}$ is expressed as

$$
r_{j, k}=\sqrt{p_{u}} x_{j, k}+\sqrt{p_{u}} \sum_{i \neq j}^{J}\left[\boldsymbol{G}_{j j}^{\dagger}\right]_{k} \boldsymbol{G}_{j i} \boldsymbol{x}_{i}+\left[\boldsymbol{G}_{j j}^{\dagger}\right]_{k} \boldsymbol{n}_{j} .
$$

From (46), the uplink instantaneous signal-to-interferenceplus-noise ratio (SINR), $\gamma_{k}$, is defined as [8]

$$
\begin{aligned}
\gamma_{k}= & \frac{p_{u}}{p_{u} \sum_{i \neq j}^{J}\left\|\left[\boldsymbol{G}_{j j}^{\dagger}\right]_{k} \boldsymbol{G}_{j i}\right\|^{2}+\left\|\left[\boldsymbol{G}_{j j}^{\dagger}\right]_{k}\right\|^{2}} \\
= & \frac{\left\|\left[\boldsymbol{G}_{j j}^{\dagger}\right]_{k}\right\|^{-2}}{\sum_{i \neq j}^{J}\left\|\frac{\left[\boldsymbol{G}_{j j}^{\dagger}\right]_{k} \boldsymbol{G}_{j i}}{\left\|\left[\boldsymbol{G}_{j j}^{\dagger}\right]_{k}\right\|}\right\|^{2}+\frac{1}{\mathrm{SNR}}} .
\end{aligned}
$$

Since $\left\|\left[\boldsymbol{G}_{j j}^{\dagger}\right]_{k}\right\|^{2}=\left[\left(\boldsymbol{G}^{H} \boldsymbol{G}\right)^{-1}\right]_{k k}$, then $\left\|\left[\boldsymbol{G}_{j j}^{\dagger}\right]_{k}\right\|^{-2} \approx X_{k}$ where $X_{k} \sim \operatorname{Gamma}\left(\theta_{f}, \Theta\right)$, and $\Theta=\left(\sum_{l=1}^{L} \beta_{j l}^{2}\right) /\left(\sum_{l=1}^{L} \beta_{j l}\right)$ as presented in Theorem 1. Then

$$
\gamma_{k} \approx \frac{X_{k}}{\sum_{i \neq j}^{J}\left\|\boldsymbol{Y}_{i}\right\|^{2}+\frac{1}{\mathrm{SNR}}}
$$

where $\boldsymbol{Y}_{i}=\left[\boldsymbol{G}_{j j}^{\dagger}\right]_{k} \boldsymbol{G}_{j i} /\left\|\left[\boldsymbol{G}_{j j}^{\dagger}\right]_{k}\right\|$.

From Theorem 1, $\boldsymbol{G}_{j i}^{H} \boldsymbol{G}_{j i}$ has approximately a complex central Wishart distribution with degrees of free$\operatorname{dom} N_{r}\left(\sum_{l=1}^{L} \beta_{i l}\right)^{2} /\left(\sum_{l=1}^{L} \beta_{i l}^{2}\right)$ and covariance matrix $\boldsymbol{\Sigma}=$ $\left(\sum_{l=1}^{L} \beta_{i l}^{2}\right) /\left(\sum_{l=1}^{L} \beta_{i l}\right) \boldsymbol{I}_{N_{t}}$ [36]. Then, conditioned on $\left[\boldsymbol{G}_{j j}^{\dagger}\right]_{k}$, $\boldsymbol{Y}_{i}$ is approximately a zero-mean complex Gaussian vector with a covariance matrix $\boldsymbol{\Sigma}_{\mathcal{I}}=\left(\sum_{l=1}^{L} \beta_{i l}^{2}\right) /\left(\sum_{l=1}^{L} \beta_{i l}\right) \boldsymbol{I}_{N_{t}}$. Therefore, $\left\|\boldsymbol{Y}_{i}\right\|^{2}$ can be defined as

$$
\left\|\boldsymbol{Y}_{i}\right\|^{2} \approx \sum_{k=1}^{N_{t}} y_{k}
$$

where $y_{k}$ is an exponential random variable with mean $\Theta_{I}=$ $\sum_{l=1}^{L} \beta_{i l}^{2} / \sum_{l=1}^{L} \beta_{i l}$.

Thus, $X_{k}$ and $\left\|\boldsymbol{Y}_{i}\right\|^{2}$ are independent non-negative random variables, and we can write eq. (48) as

$$
\gamma_{k} \approx \frac{X_{k}}{\mathcal{I}_{j}+\frac{1}{\mathrm{SNR}}}
$$

where $\mathcal{I}_{j}=\sum_{i \neq j}^{J}\left\|\boldsymbol{Y}_{i}\right\|^{2}$ represents the interference resulting from adjacent cells being shared the same frequency band.

The following lemma can be used to precisely compute the spectral efficiency of the system.

Lemma 2. [40, Eq. (5)] For $x_{1}, \ldots, x_{N}, y_{1}, \ldots, y_{M}>0$

$\ln \left(1+\frac{\sum_{n=1}^{N} x_{n}}{\sum_{m=1}^{M} y_{m}+1}\right)=\int_{0}^{\infty} \frac{1}{z}\left(1-e^{-z \sum_{n=1}^{N} x_{n}}\right) e^{-z \sum_{m=1}^{M} y_{m}} d z$

Theorem 5. For given large-scale coefficients, an approximate closed-form analytical expression for the average spectral 
efficiency of the home cell $j$ is given by

$$
\begin{aligned}
\mathcal{R}_{j}\left(\beta_{i l}\right)= & N_{t}\left(\log _{2} e\right) \sum_{i=1}^{I} \frac{\mathcal{A}_{i}}{\mathcal{B}_{i}}\left(1-\mathcal{M}\left(\beta_{j l}\right)\right) \\
& \times \prod_{i=1, i \neq j}^{J}\left(\mathcal{I}\left(\beta_{i l}\right)\right)^{N_{t}}+R_{I}
\end{aligned}
$$

where $\mathcal{M}\left(\beta_{j l}\right)=\left(\frac{1}{1+\mathcal{D}_{i} \Theta}\right)^{\theta_{f}}, \mathcal{I}\left(\beta_{i l}\right)=\frac{1}{1+\mathcal{D}_{i} \Theta_{I}}, \mathcal{D}_{i}=$ $\mathrm{SNR}_{i}, \mathcal{A}_{i}$ and $\mathcal{B}_{i}$ are the $i$ th weight and abscissa, respectively, of the $I$ th order Laguerre polynomial.

Proof: From Lemma 2, we can readily derive an exact expression for the average spectral efficiency over small-scale fading as follows

$$
\begin{aligned}
\mathcal{R}_{j}\left(\beta_{i l}\right)= & \sum_{k=1}^{N_{t}} \mathbb{E}_{X_{k}, \mathcal{I}_{j}}\left[\left(\log _{2} e\right) \int_{0}^{\infty} \frac{e^{-z / \mathrm{SNR}}}{z}\right. \\
& \left.\times\left(1-e^{-z X_{k}}\right) e^{-z \mathcal{I}_{j}} d z\right] \\
& =N_{t}\left(\log _{2} e\right) \int_{0}^{\infty} \frac{e^{-z / \mathrm{SNR}}}{z}\left(1-\mathcal{M}\left(z, \beta_{j l}\right)\right) \\
& \times \mathbb{E}_{\mathcal{I}_{j}}\left[e^{-z \mathcal{I}_{j}}\right] d z
\end{aligned}
$$

where $\mathcal{M}\left(z, \beta_{j l}\right)=\left(\frac{1}{1+z \Theta}\right)^{\theta_{f}}$.

Using the distribution of $y_{k}$, we have

$$
\begin{aligned}
\mathbb{E}_{\mathcal{I}_{j}\left[e^{-z \mathcal{I}_{j}}\right]=} \mathbb{E}_{\mathcal{I}_{j}}\left[e^{-z \sum_{i \neq j}^{J}\left\|\boldsymbol{Y}_{i}\right\|^{2}}\right] \\
=\mathbb{E}_{y_{k}}\left[e^{-z \sum_{i \neq j}^{J} \sum_{k=1}^{N_{t}} y_{k}}\right] \\
=\prod_{i=1, i \neq j}^{J} \prod_{k=1}^{K} \mathbb{E}_{y_{k}}\left[e^{-z y_{k}}\right] \\
=\prod_{i=1, i \neq j}^{J}\left(\mathcal{I}\left(z, \beta_{i l}\right)\right)^{N_{t}}
\end{aligned}
$$

where $\mathcal{I}\left(z, \beta_{i l}\right)=\frac{1}{1+z \Theta_{I}}$.

Substituting (54) into (53), we have

$$
\begin{aligned}
\mathcal{R}_{j}\left(\beta_{i l}\right)= & N_{t}\left(\log _{2} e\right) \int_{0}^{\infty} \frac{e^{-z / \mathrm{SNR}}}{z}\left(1-\mathcal{M}\left(z, \beta_{j l}\right)\right) \\
& \times \prod_{i=1, i \neq j}^{J}\left(\mathcal{I}\left(z, \beta_{i l}\right)\right)^{N_{t}} d z .
\end{aligned}
$$

In the same way, we can simplify the single integration in (55) to a finite summation using the Gauss-Laguerre quadrature method and conclude the proof.

\section{NumERICAL RESUlTS}

In this section, we validate the accuracy of our theoretical analysis presented in Sections IV, V and VI and assess the performance of the spectral efficiency against different system parameters via a set of Monte Carlo simulations. We consider D-MIMO system illustrated in Fig. 1.Here, the circular shape of the respective cell with radius $D$, and the polar coordinates system are adopted. Then, $\boldsymbol{D}_{l}=D_{l} \angle \theta_{l}$ and $\boldsymbol{\rho}=\rho \angle \theta$, and the distance $d_{l}$ is calculated using Euler's formula as [38]

$$
d_{l}=\left|D_{l}\left[\cos \left(\theta+\theta_{l}\right)+j \sin \left(\theta+\theta_{l}\right)\right]-\rho\right| .
$$

The results presented in this section are for a special topology, assuming a cell includes 5 RPs $(L=5)$, distributing over the cell at the following polar coordinates

$$
\left(D_{1}, 0\right),\left(D_{2}, 0\right),\left(D_{3}, \pi / 2\right),\left(D_{4}, \pi\right),\left(D_{5}, 3 \pi / 2\right)
$$

where

$$
D_{1}=0, D_{2}=D_{3}=D_{4}=D_{5}=0.6 D \text {. }
$$

According to this topology, one of the RPs is assumed to be at the center $(0,0)$ of the cell whereas the others are uniformly deployed on a circle of radius $D_{l \neq 1}=0.6 D$. This special topology enables us to prove the accuracy of our analysis and reveal the effects of some parameters on the system performance.

In Figs. 2-7, the impacts of various system parameters e.g., the transmit power of each antenna of the user, the number of RPs antennas, RPs sites and user locations on the spectral efficiency are investigated for the case of finite number of RPs antennas, and a comparison of spectral efficiency of the system under consideration with the spectral efficiency of the traditional C-MIMO system under the same conditions are provided as well. Also, it is important to recall that, these results have been obtained without taking the implications of shadowing fading into account. It is clearly seen that the exact results being obtained via Monte Carlo simulations of the spectral efficiency formula in (15) with the instantaneous SNR, $\gamma_{k}$, given by (11), closely match the spectral efficiency results of the proposed analytical expression in (25) when $N_{r}>N_{t}$.

In Fig. 2, the spectral efficiency of D-MIMO system with $\mathrm{ZF}$ receiver is plotted against SNR with $L=5$, and $N_{t}=10$ for different numbers of RPs antennas, $N_{r}$. As expected, the spectral efficiency increases with increasing the SNR. It is also seen that, having more RP receive antennas, $N_{r}$, improve the performance of the system through increasing the receive diversity and reducing the effect of noise enhancement. In addition, Fig. 2 evaluates the accuracy of the new results compared with the exact results (i.e., simulation results) and the approximation in [31, Eq. (12)]. It should be noticed that the shape parameter in [31, Eq. (12)] with our system parameters is $\theta_{f}=\frac{\left(\sum_{l=1}^{L} \beta_{l}\right)^{2}}{L\left(\sum_{l=1}^{L} \beta_{l}^{2}\right)}\left(L N_{r}-N_{t}+1\right)$.

Assuming all of RPs receive antennas, $L N_{r}$, for D-MIMO system under study are installed at the BS placed at the cell center of C-MIMO system, a fair comparison between D-MIMO system and C-MIMO system is obtained. Fig. 3 compares the spectral efficiency of C-MIMO system with $N_{r}=100$ at the BS with the spectral efficiency of D-MIMO system with $L=5$ and $N_{r}=20$. We keep $N_{t}=10$ for both 


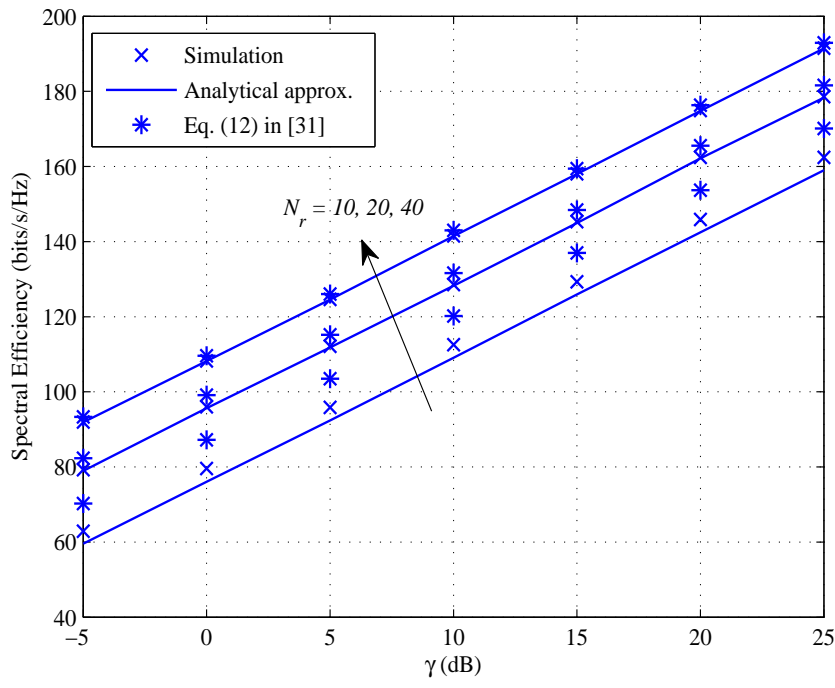

Fig. 2. Spectral efficiency against SNR for different numbers of RPs antennas $N_{r}\left(L=5, N_{t}=10, D_{l}=0.6 D, v=3\right)$.

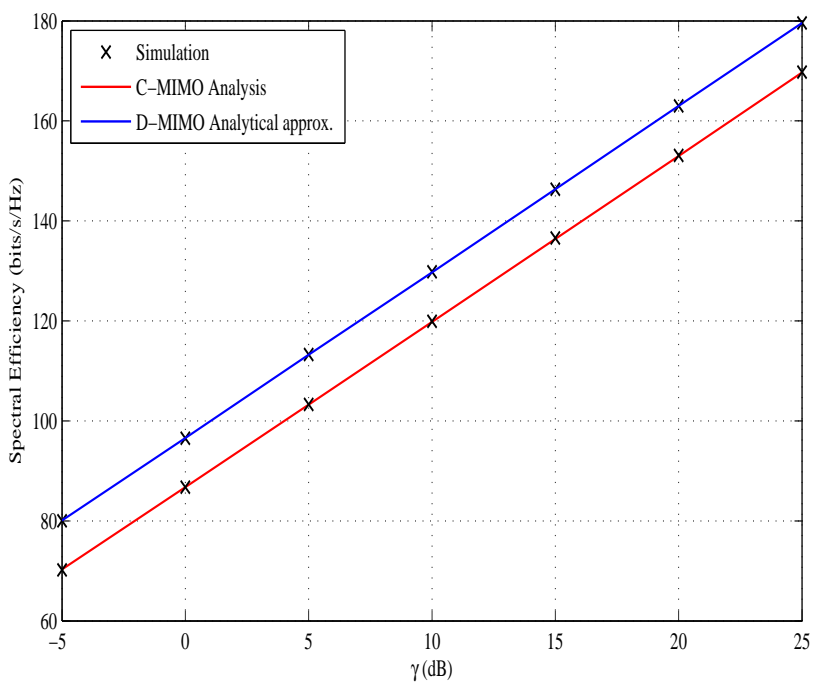

Fig. 3. Spectral efficiency against SNR for C-MIMO system ( $L=1, N_{r}=$ $100, N_{t}=10, v=3$ ) and D-MIMO system ( $L=5, N_{r}=20, N_{t}=10$, $\left.D_{l}=0.6 D, v=3\right)$.

systems. In Fig. 3, we see that the spectral efficiency for the D-MIMO setting considered is larger than that of C-MIMO system. It is also evident that, at the same performance for both systems, D-MIMO system can save about $33.33 \%$ of the power at $\gamma_{k}=5 \mathrm{~dB}$, and about $20 \%$ of the power at $\gamma_{k}=10$ dB.

In Fig. 4, the spectral efficiency against $N_{t}$ is displayed with $\gamma=15 \mathrm{~dB}$ for $N_{r}=10$ and 80 . Fig. 4 reveals that as the number of transmit antennas, $N_{t}$, increases, the spectral efficiency increases as well. For $N_{r}=10$, it is readily observed that the simulation results closely match with analytical results when $N_{t} \leq 7$. However, the gap between the simulation results and analytical results increases slightly

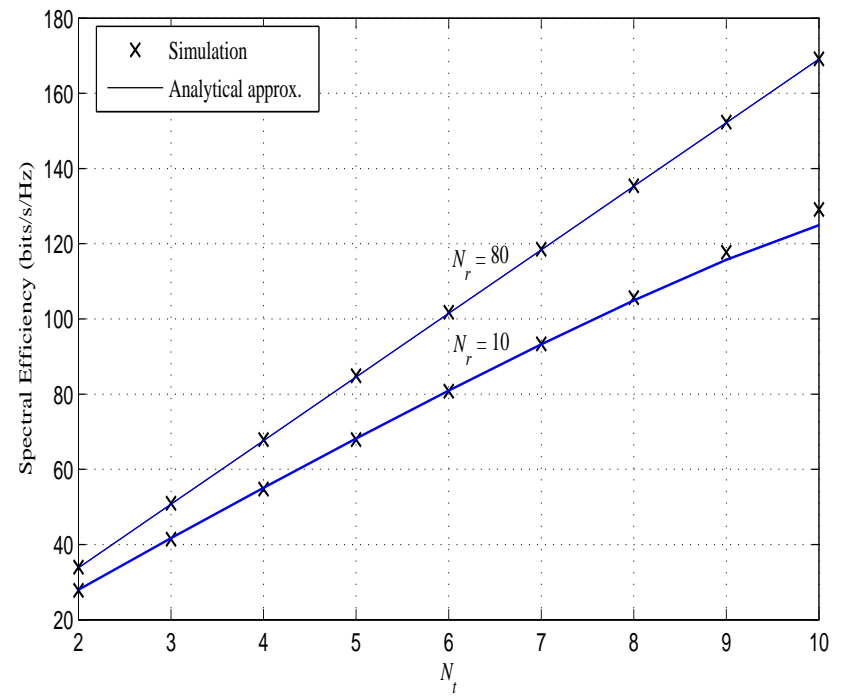

Fig. 4. Spectral efficiency against $N_{t}$ for different $N_{r}(L=5, \gamma=15 \mathrm{~dB}$, $D_{l}=0.6 D, v=3$ ).

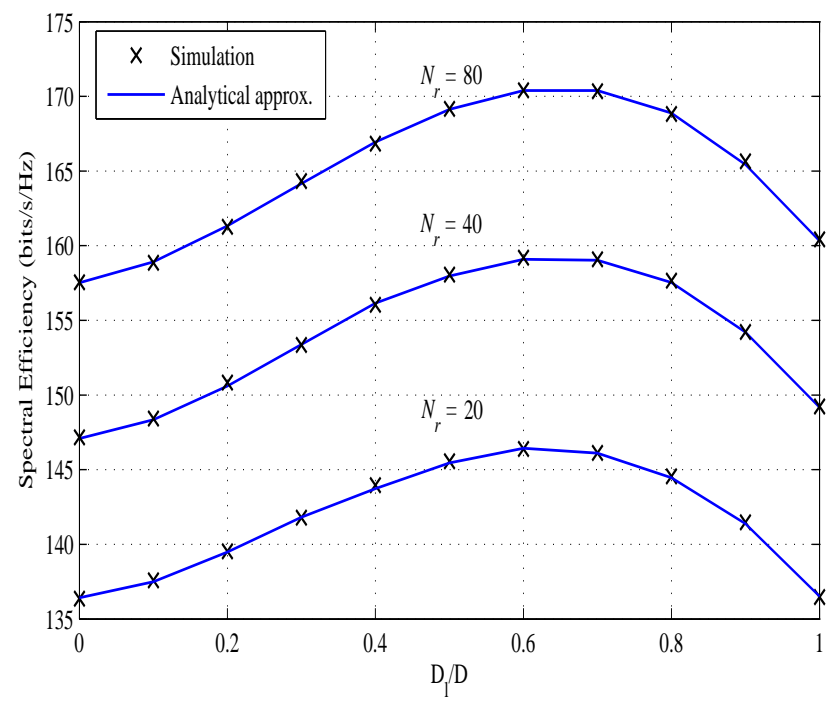

Fig. 5. Spectral efficiency against RPs positions for different $N_{r}(L=5$, $N_{t}=10, \gamma=15 \mathrm{~dB}, v=3$ ).

when $N_{t}>7$, which confirms the validity of what we have mentioned above.

The spectral efficiency of the system at $\gamma=15 \mathrm{~dB}$ is depicted against $D_{l} / D$ in Fig. 5 for various number of $N_{r}$. Fig. 5 investigates the effect of RPs locations on the spectral efficiency of the D-MIMO setting considered, and illustrates the optimal radius of the circle (i.e., $D_{l, l \neq 1}$ ), which $L-1$ of RPs are placed on, to maximize the spectral efficiency of the system. Also, it is noted that, the point at $D_{l} / D=0$ represents the C-MIMO system model. It is worth mentioning here that, the symmetrical configuration is adopted in this model. This implies all RPs except the central one are placed at the same distance to the center of the cell.

Figs. 6-7 compare the spectral efficiency of C-MIMO and 


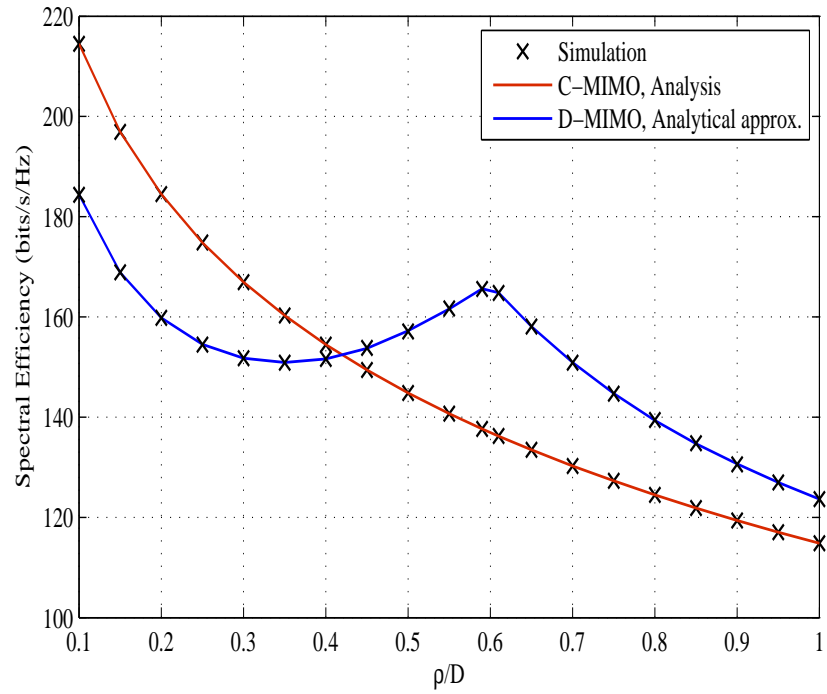

Fig. 6. Spectral efficiency against $\rho / D$ for C-MIMO system ( $L=1, N_{r}=$ $\left.100, N_{t}=10, \gamma=15 \mathrm{~dB}, v=3\right)$ and D-MIMO system $\left(L=5, N_{r}=20\right.$, $N_{t}=10, \gamma=15 \mathrm{~dB}, D_{l}=0.6 D, v=3$ ).

D-MIMO systems with respect to different user locations. The spectral efficiency for both systems against $\rho / D$ are depicted in Fig. 6 by averaging $\mathcal{R}\left(d_{l}\right)$ in (25) over $\theta$. It is observed that D-MIMO system considered yields higher spectral efficiency than C-MIMO system when $\rho$ varies from about $0.4 D$ to the cell edge. In other words, D-MIMO system offers worse spectral efficiency than C-MIMO system up to a certain point $(\rho / D \approx 0.4)$, and then outperforms the C-MIMO system. In Fig. 7, the spectral efficiency for both systems are drawn against $\theta$ via averaging the conditional $\mathcal{R}\left(d_{l}\right)$ in (25) over $\rho$. Here, the spectral efficiency of C-MIMO system is not affected by the polar angle of user, $\theta$, whereas the spectral efficiency of D-MIMO system increases as $\theta$ approaches $\theta_{l}$. We finally notice that, being the user close to one of RPs of the D-MIMO system can strongly increase the spectral efficiency.

Fig. 8 shows the spectral efficiency performance of the system against $N_{r}$. The simulated results given by (15) and (31), for the exact spectral efficiency at $\gamma=15 / N_{r} \mathrm{~dB}$ and its asymptote at $\gamma=15 \mathrm{~dB}$, respectively, exactly coincide with the spectral efficiency results of the proposed analytical expressions in (25) and (32), respectively. As anticipated, with $\gamma=15 / N_{r}$, the spectral efficiency approaches a constant value as $N_{r}$ grows large. This implies that, by using a large number of $N_{r}$, we can cut the transmit power, $\gamma, N_{r}$ times and achieve the same performance of a SISO system with transmit power $\gamma$.

In Figs. 9 and 10, the effects of shadowing fading and some system parameters e.g., RPs sites on the spectral efficiency are assessed for the case of large number of RPs antennas, $N_{r}$. We can clearly see that the analytical results based on (32) are overlaid with the outputs of a Monte Carlo simulation of (31).

The spectral efficiency is plotted in Fig. 9 against the standard deviation of the log-normal shadow fading, $\sigma$, for different $\gamma$ values. As expected, Fig. 9 shows that the spectral

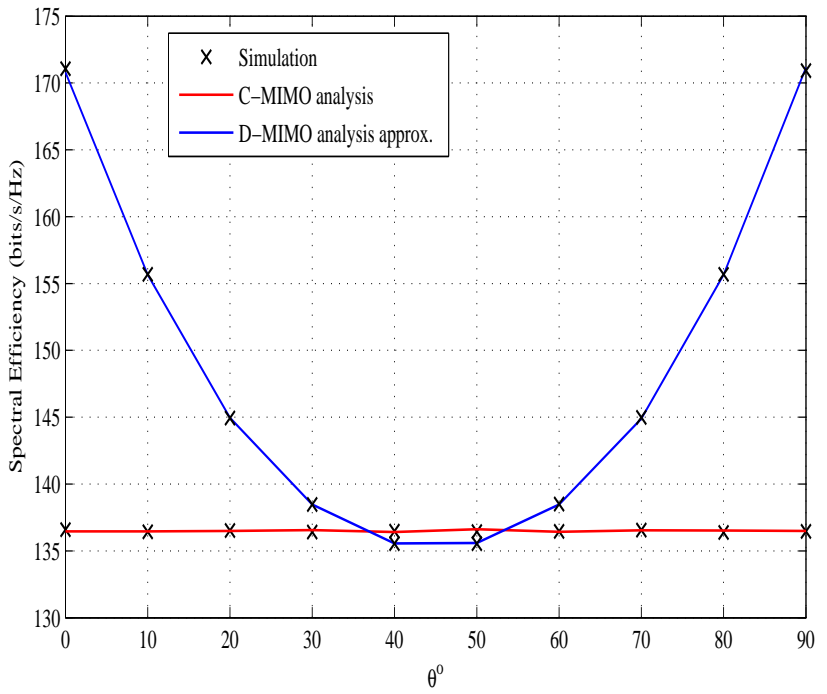

Fig. 7. Spectral efficiency against $\theta$ for C-MIMO system ( $L=1, N_{r}=100$, $N_{t}=10, \gamma=15 \mathrm{~dB}, v=3$ ) and D-MIMO system ( $L=5, N_{r}=20$, $\left.N_{t}=10, \gamma=15 \mathrm{~dB}, D_{l}=0.6 D, v=3\right)$.

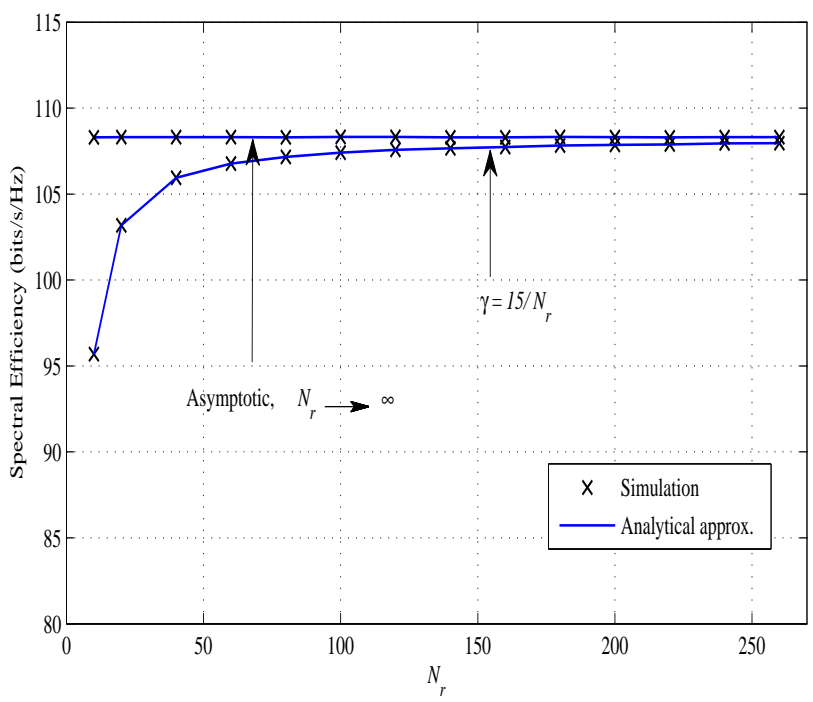

Fig. 8. Spectral efficiency (exact and asymptotic) against $N_{r}(L=5$, $N_{t}=10, D_{l}=0.6 D, v=3$ ).

efficiency almost increases linearly with $\sigma$ regardless of $\gamma$ values.

Comparing the lower bound on the asymptotic spectral efficiency in (37) with the average asymptotic spectral efficiency in (31) and 32) is provided in Fig. 10. It can be observed that the lower bound remains quite close to the exact spectral efficiency results for over the entire $\gamma$ range.

The spectral efficiency per cell of a multi-cell system with $J=4$ is plotted against $\gamma$ in Fig. 11 for various number of $N_{r}$, assuming $\beta_{j 1}=0.2, \beta_{j 2}=0.3, \beta_{j 3}=0.4, \beta_{j 4}=0.5$, and $\beta_{j 5}=0.6$ for the respective cell $j$, and $\beta_{i 1}=0.05$, $\beta_{i 2}=0.1, \beta_{i 3}=0.15, \beta_{i 4}=0.2$, and $\beta_{i 5}=0.25$ for the $i$ th co-channel interferer cell. Fig. 11 illustrates the effect of cochannel interference on the spectral efficiency of the system. 


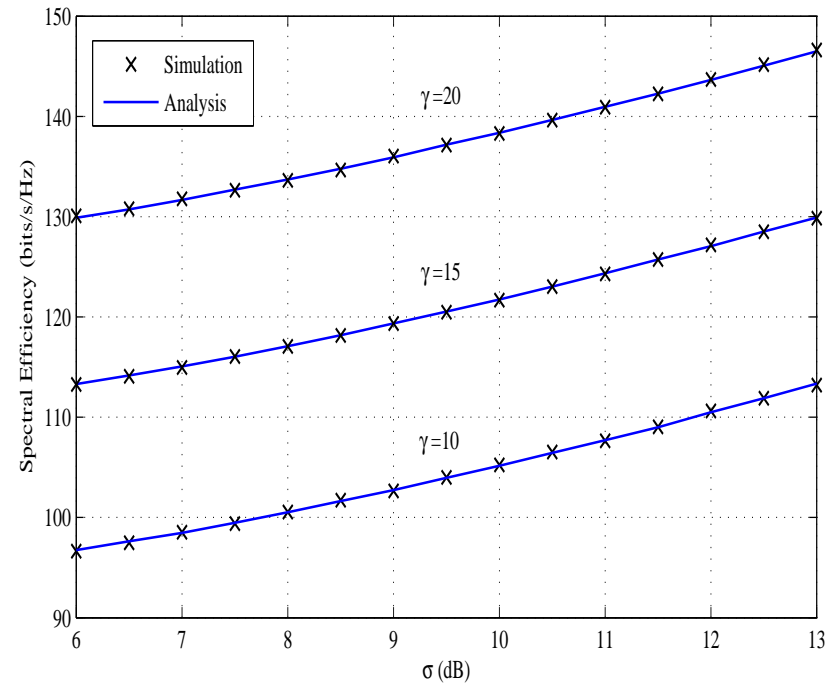

Fig. 9. Spectral efficiency against the standard deviation of log-normal shadow fading $\left(L=5, N_{r} \rightarrow \infty, N_{t}=10, D_{l}=0.6 D, v=3\right)$.

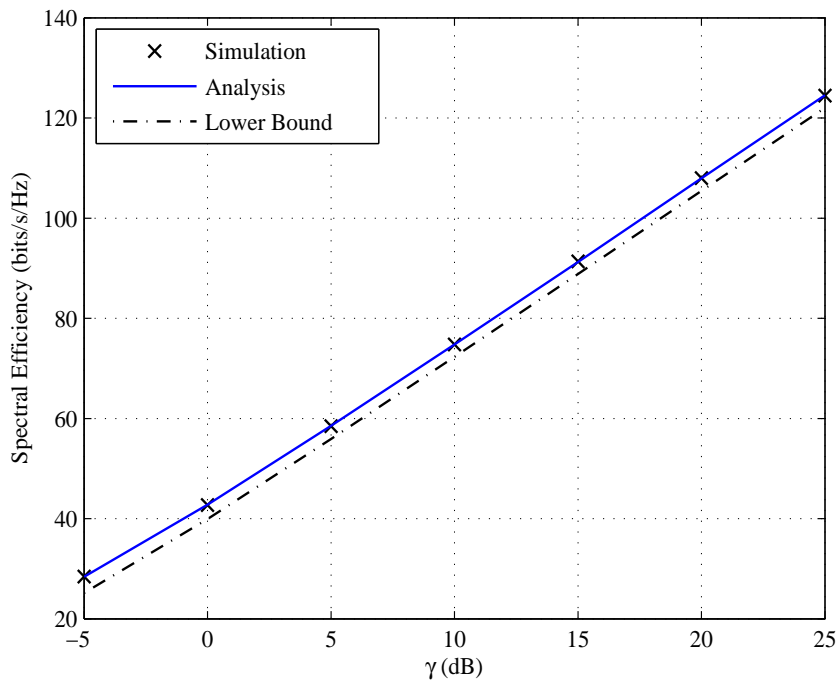

Fig. 10. Spectral efficiency ( exact and lower bound ) against SNR ( $L=5$, $\left.N_{r} \rightarrow \infty, N_{t}=10, D_{l}=0.6 D, \rho=D, \theta=\pi / 4, v=3\right)$.

It is easily observed that the analytical approximation results being obtained via (52) is quite accurate with respect to the exact results being generated via Monte Carlo simulations using (14) with the instantaneous SNR, $\gamma_{k}$, given by (47). As anticipated with the out-of-cell interference, the spectral efficiency improves by increasing $N_{r}$, whereas we can not improve the spectral efficiency of the system by increasing the transmit power of each user because the interference power increases as well.

\section{CONCLUSION}

In this paper, we have elaborated on the spectral efficiency analysis of D-MIMO systems with ZF receivers. As an initial step, we have presented a statistical characterization of $\mathrm{ZF}$

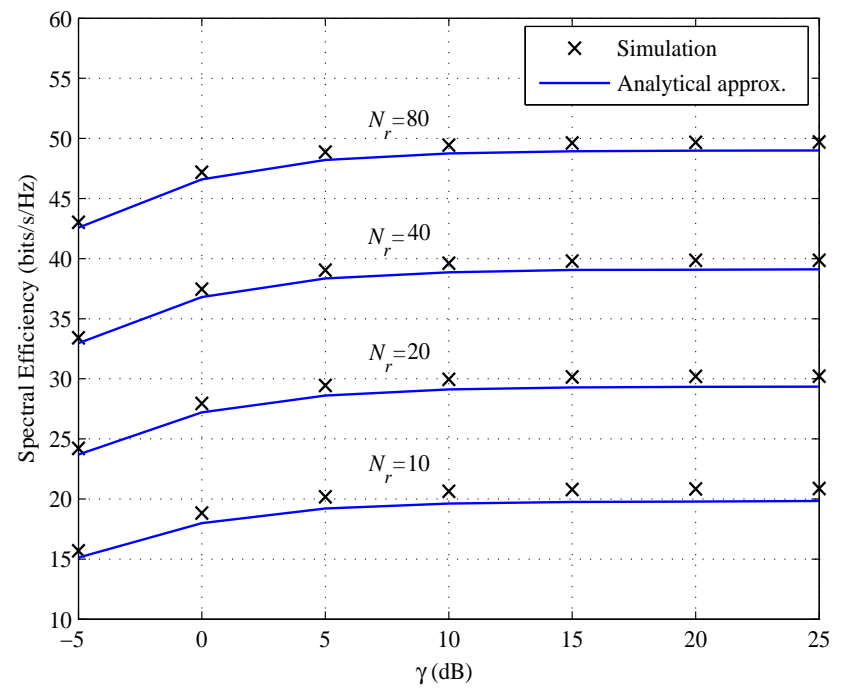

Fig. 11. Spectral efficiency against SNR for different $N_{r}(J=4, L=5$, $\left.N_{t}=10\right)$.

receivers over a single-cell D-MIMO channels. Then, an accurate approximation for the PDF of the instantaneous SNR for the system has been performed. With fixed large-scale fading, we have derived a new precise expression for the spectral efficiency of the system as a function of model parameters e.g., $\gamma$, user location and number of antennas. This expression enabled us to compare the performance of the system under investigation with C-MIMO systems, and quantify the effects on the spectral efficiency as that parameters vary. In addition, we have examined the spectral efficiency in the asymptotically very large number of RPs antennas, $N_{r}$, regime.Taking the average over shadow fading, new exact expression for the spectral efficiency has been provided and useful insights into the impact of shadowing on the system performance have been obtained under practical environments. In parallel, a closedform lower bound on the asymptotic spectral efficiency has also been offered, which has been a quite tight over the entire $\gamma$ range. We have further worked on the multi-cell system and the effect of co-channel interference on the spectral efficiency.

\section{REFERENCES}

[1] P. Li, D. Paul, R. Narasimhan, and J. Cioffi, "On the distribution of SINR for the MMSE MIMO receiver and performance analysis," IEEE Trans. Inf. Theory, vol. 52, no. 1, pp. 271-286, Jan. 2006.

[2] C.-J. Chen and L.-C. Wang, "Performance analysis of scheduling in multiuser MIMO systems with zero-forcing receivers," IEEE J. Sel. Topics Signal Process., vol. 25, no. 7, pp. 1435-1445, Sep. 2007.

[3] N. Kim, Y. Lee, and H. Park, "Performance analysis of MIMO system with linear MMSE receiver," IEEE Trans. Wireless Commun., vol. 7, no. 11 , pp. 4474-4478, Nov. 2008.

[4] M. McKay, I. Collings, and A. Tulino, "Achievable sum rate of MIMO MMSE receivers: A general analytic framework," IEEE Trans. Inf. Theory, vol. 56, no. 1, pp. 396-410, Jan. 2010.

[5] F. Rusek, D. Persson, B. K. Lau, E. Larsson, T. Marzetta, O. Edfors, and F. Tufvesson, "Scaling up MIMO: Opportunities and challenges with very large arrays," IEEE Signal Process. Mag., vol. 30, no. 1, pp. 40-60, Jan. 2013.

[6] H. Q. Ngo, E. Larsson, and T. Marzetta, "Energy and spectral efficiency of very large multiuser MIMO systems," IEEE Trans. Commun., vol. 61, no. 4, pp. 1436-1449, Apr. 2013. 
[7] H. Ngo, E. Larsson, and T. Marzetta, "The multicell multiuser MIMO uplink with very large antenna arrays and a finite-dimensional channel," IEEE Trans. Commun., vol. 61, no. 6, pp. 2350-2361, Jun. 2013.

[8] H. Q. Ngo, M. Matthaiou, T. Duong, and E. Larsson, "Uplink performance analysis of multicell MU-SIMO systems with ZF receivers," IEEE Trans. Vehicular Tech., vol. 62, no. 9, pp. 4471-4483, Nov. 2013.

[9] A. A. Saleh, A. J. Rustako, and R. S. Roman, "Distributed antennas for indoor radio environment," IEEE Trans. Commun., vol. 35, no. 12, pp. 1245-1252, Dec. 1987.

[10] M. Clark, I. Willis, T., L. Greenstein, J. Rustako, A., V. Erceg, and R. Roman, "Distributed versus centralized antenna arrays in broadband wireless networks," in Proc. 2001 IEEE VTC, vol. 1, pp. 33-37 vol.1.

[11] H. Xia, A. Herrera, S. Kim, and F. Rico, "A CDMA-distributed antenna system for in-building personal communications services," IEEE J. Sel. Areas in Commun., vol. 14, no. 4, pp. 644-650, May 1996.

[12] K. Kerpez, "A radio access system with distributed antennas," IEEE Trans. Vehicular Tech., vol. 45, no. 2, pp. 265-275, May 1996.

[13] W. Roh and A. Paulraj, "Outage performance of the distributed antenna systems in a composite fading channel," in Proc. 2002 IEEE VTC, vol. 3, pp. 1520-1524 vol.3.

[14] H. Zhuang, L. Dai, L. Xiao, and Y. Yao, "Spectral efficiency of distributed antenna system with random antenna layout," Electron. Lett., vol. 39, no. 6, pp. 495-496, Mar. 2003.

[15] L. Dai, S. Zhou, and Y. Yao, "Capacity analysis in CDMA distributed antenna systems," IEEE Trans. Wireless Commun., vol. 4, no. 6, pp. 2613-2620, Nov. 2005.

[16] W. Choi and J. Andrews, "Downlink performance and capacity of distributed antenna systems in a multicell environment," IEEE Trans. Wireless Commun., vol. 6, no. 1, pp. 69-73, Jan. 2007.

[17] J. Andrews, W. Choi, and R. Heath, "Overcoming interference in spatial multiplexing MIMO cellular networks,” IEEE Trans. Wireless Commun., vol. 14, no. 6, pp. 95-104, Dec. 2007.

[18] L. Dai, "A comparative study on uplink sum capacity with co-located and distributed antennas," IEEE J. Sel. Areas in Commun., vol. 29, no. 6, pp. 1200-1213, Jun. 2011.

[19] X. Wang, P. Zhu, and M. Chen, "Antenna location design for generalized distributed antenna systems," IEEE Commun. Lett., vol. 13, no. 5, pp. 315-317, May 2009.

[20] Y. Qian, M. Chen, X. Wang, and P. Zhu, "Antenna location design for distributed antenna systems with selective transmission," in Proc. 2009 Intern. Conf.WCSP, Nov., pp. 1-5.

[21] E. Park and I. Lee, "Antenna placement for downlink distributed antenna systems with selection transmission," in Proc. 2011 IEEE VTC, May, pp. $1-5$.

[22] E. Park, S.-R. Lee, and I. Lee, "Antenna placement optimization for distributed antenna systems," IEEE Trans. Wireless Commun., vol. 11, no. 7, pp. 2468-2477, July 2012.

[23] H. Zhang and H. Dai, "On the capacity of distributed MIMO systems," in Proc. Conf. Inform. Sciences and Systems, Mar. 2004.

[24] H. Dai, "Distributed versus co-located MIMO systems with correlated fading and shadowing," in Proc. 2006 IEEE ICASSP, vol. 4, May, pp. IV-IV.

[25] H. Dai, H. Zhang, and Q. Zhou, "Some analysis in distributed MIMO systems," J. Commun., vol. 2, no. 3, 2007.

[26] F. Heliot, R. Hoshyar, and R. Tafazolli, "An accurate closed-form approximation of the distributed MIMO outage probability," IEEE Trans. Wireless Commun., vol. 10, no. 1, pp. 5-11, Jan. 2011.

[27] S.-R. Lee, S.-H. Moon, J.-S. Kim, and I. Lee, "Capacity analysis of distributed antenna systems in a composite fading channel," IEEE Trans. Wireless Commun., vol. 11, no. 3, pp. 1076-1086, Mar. 2012.

[28] D. Wang, J. Wang, X. You, Y. Wang, M. Chen, and X. Hou, "Spectral efficiency of distributed MIMO systems," IEEE J. Sel. Areas in Commun., vol. 31, no. 10, pp. 2112-2127, Oct. 2013.

[29] X. Li, L. Li, X. Su, Z. Wang, and P. Zhang, "Approximate capacity analysis for distributed MIMO system over generalized-K fading channels," in Proc. 2015 IEEE WCNC, pp. 235-240.

[30] R. Senanayake, P. Smith, P. L. Yeoh, and J. Evans, "An SNR approximation for distributed massive MIMO with zero forcing," IEEE Commun. Lett., vol. 19, no. 11, pp. 1885-1888, 2015.

[31] K. Hosseini, W. Yu, and R. S. Adve, "Large-scale MIMO versus network MIMO for multicell interference mitigation," IEEE J. Sel. Topics in Signal Process., vol. 8, no. 5, pp. 930-941, 2014.

[32] M. Park, C.-B. Chae, and R. Heath, "Ergodic capacity of spatial multiplexing MIMO systems with ZF receivers for log-normal shadowing and Rayleigh fading channels," in Proc. 2007 IEEE PIMRC, Sep., pp. $1-5$.

[33] R. Heath, T. Wu, Y. H. Kwon, and A. Soong, "Multiuser MIMO in distributed antenna systems with out-of-cell interference," IEEE Trans. Signal Process., vol. 59, no. 10, pp. 4885-4899, 2011.

[34] M. Matthaiou, N. Chatzidiamantis, G. Karagiannidis, and J. Nossek, "ZF detectors over correlated k fading MIMO channels," IEEE Trans. Commun., vol. 59, no. 6, pp. 1591-1603, Jun. 2011

[35] M. Matthaiou, C. Zhong, M. McKay, and T. Ratnarajah, "Sum rate analysis of ZF receivers in distributed MIMO systems," IEEE J. Sel. Areas in Commun., vol. 31, no. 2, pp. 180-191, Feb. 2013.

[36] D. Nel and C. Merwe, "A solution to the multivariate Behrens-Fisher problem," Commun. Stat. - Theory Methods, vol. 15, pp. 3719-3735, 1986.

[37] D. Gore, R. Heath, and A. Paulraj, "Transmit selection in spatial multiplexing systems," IEEE Commun. Lett., vol. 6, no. 11, pp. 491-493, Nov. 2002.

[38] M. M. Aldosari and K. A. Hamdi, "Performance analysis of energy efficient distributed antenna systems," in Proc. 2015 IEEE VTC Spring, May, pp. 1-5.

[39] K. Hamdi, "Capacity of MRC on correlated Rician fading channels," IEEE Trans. Commun., vol. 56, no. 5, pp. 708-711, May 2008

[40] — - "A useful lemma for capacity analysis of fading interference channels," IEEE Trans. Commun., vol. 58, no. 2, pp. 411-416, Feb. 2010.

[41] M. Abramowitz and I. Stegun, "Handbook of mathematical functions:with formulas and mathematical tables," New York:Dover, 1965. 